\title{
Superparamagnetic Iron Oxide Nanoparticles
} Protect Human Gingival Fibroblasts from Porphyromonas gingivalis Invasion and Inflammatory Stimulation

\author{
Yulian Chen ${ }^{1,2}$ \\ Qian Zhang' \\ Xuan Qin' \\ Jin $\mathrm{Li}^{1}$ \\ Yantao Zhao ${ }^{3,4}$ \\ Yang Xia' \\ 'Jiangsu Key Laboratory of Oral \\ Diseases, Nanjing Medical University, \\ Nanjing, Jiangsu, People's Republic of \\ China; ${ }^{2}$ The Affiliated Stomatological \\ Hospital of Soochow University, Suzhou \\ Stomatological Hospital, Suzhou, Jiangsu, \\ People's Republic of China; ${ }^{3}$ Institute of \\ Orthopedics, Fourth Medical Center of \\ the General Hospital of CPLA, Beijing, \\ People's Republic of China; ${ }^{4}$ Beijing \\ Engineering Research Center of \\ Orthopedics Implants, Beijing, People's \\ Republic of China
}

Correspondence: Yang Xia Jiangsu Key Laboratory of Oral Diseases, Nanjing Medical University, Nanjing, Jiangsu, 210029, People's Republic of China

Tel +86 25-52283229

Fax +86-25-86516414

Email xiayang@njmu.edu.cn

Yantao Zhao

Institute of Orthopedics, Fourth Medical Center of the General Hospital of CPLA, Beijing, 100048, People's Republic of China $\mathrm{Fax}+86-10-66848865$

Email 45828016@qq.com
Introduction: Modulating the inflammatory response of human gingival fibroblasts (hGFs) is important for the control of periodontal inflammation because it is a key event in the pathogenesis of periodontitis. Here, we aimed to determine whether polyglucose sorbitol carboxymethyl ether (PSC)-coated superparamagnetic iron oxide nanoparticles (SPIONs) protect hGFs against invasion and inflammatory stimulation by Porphyromonas gingivalis (P. gingivalis).

Methods: First, we determined the cytotoxicity and antimicrobial activity of PSC-SPIONs. Then, their effects on invasion of hGFs by $P$. gingivalis were evaluated by counting invading $P$. gingivalis, fluorescence staining, and transmission electron microscopy. The effect of PSCSPIONs on inflammation in hGFs induced by $P$. gingivalis lipopolysaccharide was evaluated by measurement of reactive oxygen species (ROS), and enzyme-linked immunosorbent assays, quantitative reverse transcription-polymerase chain reaction, and Western blotting of key indicator molecules. The effects of dimercaptosuccinic acid (DMSA)-coated SPIONs and the free form of PSC alone were also tested and compared with those of PSC-SPIONs. Results: PSC-SPIONs $(25 \mu \mathrm{g} / \mathrm{mL})$ are cytocompatible with hGFs and exhibit no antimicrobial effects on $P$. gingivalis. However, they inhibit invasion of hGFs by $P$. gingivalis at $15 \mu \mathrm{g} / \mathrm{mL}$. They also decrease ROS production and inflammatory cytokine secretion by hGFs at 5,15 , and $25 \mu \mathrm{g} / \mathrm{mL}$, by downregulating activation of the nuclear factor-kappa B signaling pathway. Furthermore, PSC alone does not inhibit inflammation, while DMSA-SPIONs do. This indicates that the nanosize effects of PSC-SPIONs, rather than their coating material, play the dominant role in their anti-inflammatory activity.

Conclusion: PSC-SPIONs protect hGFs against $P$. gingivalis invasion and inflammatory stimulation. Thus, they have potential for clinical application in control of periodontal inflammation.

Keywords: iron oxide nanoparticle, Porphyromonas gingivalis, human gingival fibroblast, invasion, inflammation

\section{Introduction}

Periodontitis is a bacterial inflammatory disease of periodontal tissue characterized by progressive periodontal attachment loss, alveolar bone resorption, tooth mobility, and tooth loss. ${ }^{1}$ A national health and nutrition survey conducted in the USA in 2020 revealed that the prevalence of total periodontitis in dentate adults aged 30-79 years 


\section{Graphical Abstract}

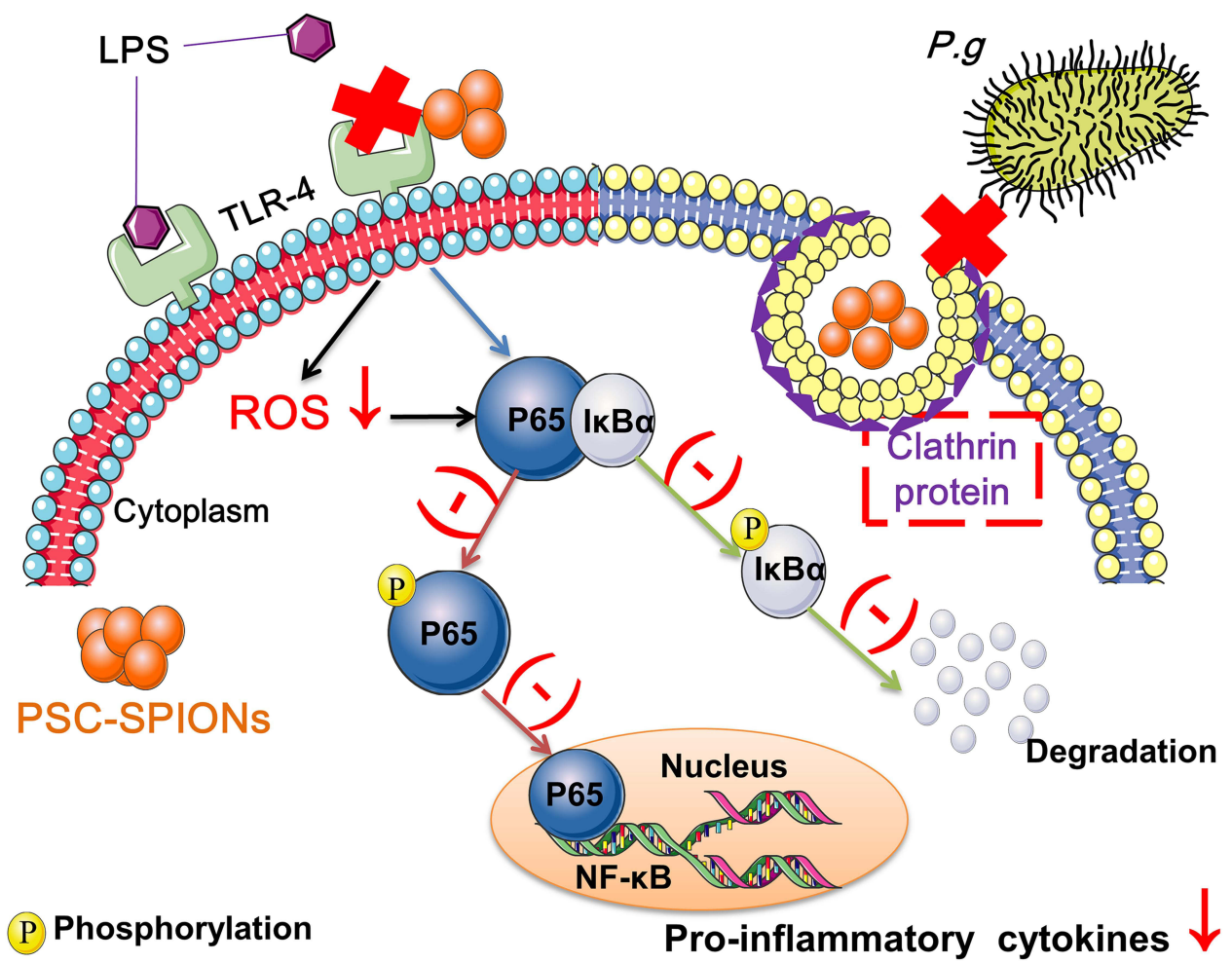

was $42.2 \pm 1.4 \%{ }^{2}$ Periodontitis is associated with systemic inflammatory conditions such as cardiovascular diseases, diabetes, and rheumatoid arthritis. ${ }^{3-5}$ Gingival inflammation is a prerequisite for the pathogenesis of periodontitis. ${ }^{6}$ Therefore, management of gingival inflammation is an important strategy for treating and preventing periodontitis as well as maintaining overall health.

Gingival inflammation presents as redness, swelling, and an increased likelihood of bleeding upon gentle probing. It starts with an unchecked immune response by gingival epithelial cells and the neutrophil barrier under microbial plaque-mediated stimulation, which is followed by an extended inflammatory response in the deeper gingival body. ${ }^{7}$

Human gingival fibroblasts (hGFs) are the most abundant cells in gingival connective tissue and play an important role in the development and persistence of periodontal inflammation. ${ }^{8}$ They are often affected by periodontal pathogenic bacteria and their lipopolysaccharides (LPSs). When stimulated by such factors, hGFs secrete cytokines and chemokines such as interleukin (IL)-6 and IL-8, which trigger inflammatory responses and exacerbate inflammation. ${ }^{9}$
Furthermore, inflammatory mediators released by hGFs can activate osteoclastic cells and induce alveolar bone resorption. $^{10}$ Therefore, modulating the inflammatory response of hGFs is crucial for controlling periodontal inflammation.

Porphyromonas gingivalis ( $P$. gingivalis) is a Gramnegative anaerobe regarded as a red-complex periodontal pathogenic bacterium that is frequently detected in deep periodontal pockets, especially in sites affected by disease. ${ }^{11}$ Invasion and subsequent immune stimulation by LPS are the two major events by which $P$. gingivalis mediates damage to hGFs. ${ }^{12}$ The entry into hGFs protects $P$. gingivalis from the phagocytes and antibodies of the host immune system, allowing it to remain viable, replicate, and spread to neighboring cells. ${ }^{13}$ Furthermore, $P$. gingivalis LPS increases the production of intercellular reactive oxygen species (ROS) and activates the nuclear factor-kappa $\mathrm{B}(\mathrm{NF}-\kappa \mathrm{B})$ pathway by upregulating toll-like receptor-2/4 (TLR-2/4), ${ }^{14}$ causing periodontal tissue injury. Thus, inhibition of $P$. gingivalis invasion and inflammatory stimulation is a viable strategy for reducing the inflammatory response of hGFs. 
Currently available methods do not effectively reduce invasion and inflammatory stimulation by $P$. gingivalis in hGFs. Scaling and root planning (SRP) by removing bacterial biofilms, calculus, and toxins from periodontally involved root surfaces is regarded as the "gold standard" of periodontal treatment, and is widely used to control microbial periodontal infection. ${ }^{15}$ However, manual operations on deep and complex diseased sites, such as furcation and interproximal areas, have limited success. ${ }^{15}$ Adjunctive systemic and local antibiotics in combination with SRP yield better results than SRP alone, more effectively decreasing probing depth and increasing the clinical attachment level, ${ }^{16}$ which indicate improved control of periodontal inflammation. However, drawbacks associated with bacterial drug resistance; side-effects such as headache, fever, vomiting, asthma, and allergic reactions; and potential effects on the gastrointestinal microbiome, limit the use of this approach. ${ }^{16}$ Therefore, alternative strategies that avoid the drawbacks of antibiotics and effectively reduce $P$. gingivalis-mediated inflammatory response in hGFs are of great clinical interest.

A promising candidate strategy is the use of superparamagnetic iron oxide nanoparticles (SPIONs). SPIONs are biosafe and have been widely applied in biomedicine. ${ }^{17}$ Furthermore, polyglucose sorbitol carboxymethyl ether (PSC)-coated $\gamma \mathrm{Fe}_{2} \mathrm{O}_{3}$ nanoparticles (PSCSPIONs) have been approved by the US Food and Drug Administration for iron replacement therapy. ${ }^{18}$ Our group has prepared PSC-SPIONs with the same structure as this nanodrug. Our PSC-SPIONs are used as a magnetic resonance imaging (MRI) T2 contrast agent to label neural stem cells. ${ }^{19}$ When applied in tissue engineering, they enhance the osteogenic differentiation of bone marrow stem cells $(\mathrm{BMSCs})^{20}$ and dental pulp stem cells. ${ }^{21}$ Moreover, our PSC-SPIONs exhibit anti-inflammatory and immunomodulatory effects and have been demonstrated to alleviate inflammation in LPS-induced sepsis and liver injury by preventing inflammatory cell infiltration and enhancing IL-10-producing macrophages; ${ }^{22}$ they decrease $\mathrm{Fe}^{3+}$-induced ROS increase in MC3T3-E1 and RAW 264.7 cells by activating the Nrf2-HO-1 pathway, ${ }^{23}$ and polyethylene glycol (PEG)-coated SPIONs decrease Escherichia coli O111:B4 LPS-induced IL-6 and tumor necrosis factor-alpha (TNF- $\alpha$ ) secretion as well as iNOS expression at the mRNA and protein levels in mouse RAW264.7 and human THP-1-derived macrophages. This decrease is positively correlated with downregulated expression of TLR- $4 .^{24}$ Furthermore, oleic acid and amphiphilic polymer-coated SPIONs suppress E. coli O111:B4 LPS-induced NF-кB activation and proinflammatory cytokine production in primary human monocytes. ${ }^{25}$ Moreover, PSC-SPIONs are superparamagnetic and can be magnetically targeted to specific diseased sites that are unreachable by SRP. Therefore, PSC-SPIONs have the potential to decrease $P$. gingivalis-mediated inflammatory responses in hGFs.

In the present study, PSC-SPIONs were used to pretreat hGFs and their effects on $P$. gingivalis invasion of hGFs and $P$. gingivalis LPS-induced ROS production and inflammatory response therein were evaluated. Furthermore, the effects of PSC-SPIONs on the NF-kBp65 pathway were detected.

\section{Materials and Methods Isolation and Purification of hGFs}

hGFs were obtained from distal gingival tissue during the extraction of the third mandibular molar from patients aged 18 to 25 years at the Stomatological Hospital Affiliated to Nanjing Medical University. Briefly, the gingival tissues were stored in Dulbecco's modified Eagle's medium (4.5 g/L D-Glucose, H-DMEM; Gibco, USA) with $2 \%(v / v)$ fetal bovine serum (FBS; ScienCell, USA) and $2 \%(v / v)$ penicillin/streptomycin (P/S; Gibco, USA). After 3 rinses with $1 \times$ phosphate-buffered saline (PBS; HyClone, USA) containing $5 \% \mathrm{P} / \mathrm{S}$, the tissues were cut into smaller pieces and digested with $3 \mathrm{~g} / \mathrm{L}$ collagenase I (Sigma-Aldrich, USA) for $30 \mathrm{~min}$ at $37{ }^{\circ} \mathrm{C}$ in a $5 \% \mathrm{CO}_{2}$ incubator. ${ }^{26}$ The tissue blocks were then cultured in H-DMEM containing $10 \% \mathrm{FBS}$ and $2 \% \mathrm{P} / \mathrm{S}$. hGFs that had been passaged three to eight times were used in this study. The cells were cultured in H-DMEM containing $10 \%$ FBS without $\mathrm{P} / \mathrm{S}$.

The experimental protocol was approved by the Stomatological College of Nanjing Medical University (Number PJ2019-064-001). Informed consent was obtained from the patients, and the guidelines of the Declaration of Helsinki were followed.

\section{Cytotoxicity of PSC-SPIONs in hGFs}

PSC-SPIONs comprising an iron oxide core and a PSC shell were obtained from Jiangsu Key Laboratory for Biomaterials and Devices of Southeast University (China). The preparation and characterization of PSCSPIONs has been described in detail previously. ${ }^{27}$ Here, the magnetic properties of PSC-SPIONs were tested using 
a superconducting quantum interference device (SQUID) magnetometer (Quantum Design MPMS-3, Quantum Design, USA) at $300 \mathrm{~K} ; 9.1 \mathrm{mg}$ of dried PSC-SPIONs were characterized under an external magnetic field in the range $\pm 30,000$ Oe. The saturation magnetization values were normalized to the mass of nanoparticles to yield the specific value of magnetization in $\mathrm{emu} / \mathrm{g}$.

The viability of hGFs treated with PSC-SPIONs (abbreviated to P-S in the group labels) was quantified using Cell Counting Kit-8 (CCK-8, DOJINDO, Japan). Briefly, hGFs were seeded in a 96-well plate at 5000 cells/well and incubated overnight. PSC-SPIONs were then added to the wells at final concentrations of 5, 15, 25,50 , and $100 \mu \mathrm{g} / \mathrm{mL}$. After incubation for 24,48 , or 72 h, $10 \%$ CCK-8 solution in culture medium was added into each well. The optical density at $450 \mathrm{~nm}\left(\mathrm{OD}_{450} \mathrm{~nm}\right)$ was determined using a microplate reader (SpectraMax $\mathrm{M}^{2}$, USA) after incubation for $2 \mathrm{~h}$.

\section{Antibacterial Properties of PSC-SPIONs Against $P$. gingivalis}

P. gingivalis (ATCC 337441; abbreviated to P. $g$ in the group labels) was cultured on brain-heart infusion (BHI, OXOID, UK)-agar plates supplemented with $5 \%$ sterile defibrinated sheep blood (DENING BIO, China), $0.5 \mathrm{mg}$ / $\mathrm{mL}$ hemin (Aladdin, China), and $0.1 \mathrm{mg} / \mathrm{mL}$ vitamin $\mathrm{K}$ (Aladdin, China) in an anaerobic incubator $\left(90 \% \mathrm{~N}_{2}, 5 \%\right.$ $\mathrm{H}_{2}, 5 \% \mathrm{CO}_{2}, 37{ }^{\circ} \mathrm{C}$ ). The bacterial concentration was established using a turbidimeter (BZ-TDR100, China). $P$. gingivalis cultures were diluted to the required concentration with cell culture medium.

The viability of $P$. gingivalis after treatment with 25 $\mu \mathrm{g} / \mathrm{mL}$ PSC-SPIONs was determined by counting the surviving colonies with untreated $P$. gingivalis as the control. Briefly, P. gingivalis was co-cultured with $25 \mu \mathrm{g} / \mathrm{mL}$ PSCSPIONs diluted in cell culture medium for $2 \mathrm{~h}$ (P-S-treated $P . g)$. The bacteria were then washed in $1 \times$ PBS three times, serially diluted, and inoculated onto BHI-agar plates. After incubation for 10 days, the colony numbers were counted. P. gingivalis treated with PSC-SPIONs were also prepared for scanning electron microscopy observation (SEM; JEOL JSM-7900F, Japan).

\section{Activity of PSC-SPIONs Against Invasion of hGFs by $P$. gingivalis}

The effects of PSC-SPIONs on the invasion of hGFs by $P$. gingivalis were investigated. hGFs in the PSC-SPIONs group were pretreated with 5,15 , or $25 \mu \mathrm{g} / \mathrm{mL}$ PSCSPIONs for $6 \mathrm{~h}$. Untreated hGFs were used as the control. The experimental groups were named 5-P-S $+P$. $g, 15-\mathrm{P}-\mathrm{S}$ $+P . g, 25-\mathrm{P}-\mathrm{S}+P . g$, and hGF $+P . g$, respectively. The hGFs were infected with $P$. gingivalis with a multiplicity of infection (MOI) of 500 for $2 \mathrm{~h}$. After rinsing off unattached $P$. gingivalis with $1 \times$ PBS three times, the hGFs were lysed using $1 \mathrm{~mL}$ sterile water for $1 \mathrm{~h}$. The lysates were serially diluted, inoculated onto BHI-agar plates, and incubated for 10 days for cell counting (in colony-forming units $(\mathrm{CFU}) / \mathrm{mL})$. A concentration of $15 \mu \mathrm{g} / \mathrm{mL}$ (15-P-S) was selected for subsequent tests.

For confocal laser scanning microscopy (CLSM) observation, $P$. gingivalis was incubated with the fluorescent probe SYTO-9 (Invitrogen, USA) in the dark at room temperature for $15 \mathrm{~min}$. Then, P. gingivalis and hGFs were coincubated for $2 \mathrm{~h}$, and the hGFs were washed twice with PBS and fixed with 4\% paraformaldehyde for $10 \mathrm{~min}$. They were then permeated with $0.1 \%$ Triton- 100 for $10 \mathrm{~min}$ and incubated with $1 \%$ bovine serum albumin (Beyotime, China) for 20-30 min. The cytoskeleton was stained using Alexa Fluor ${ }^{\circledR} 594$ Phalloidin (YEASEN, China) and the nucleus was counter-stained with 4',6-diamidino-2-phenylindole (DAPI; Beyotime, China). The hGFs were visualized by CLSM (ZEISS LSM710, Germany).

hGFs treated with $P$. gingivalis were also prepared for transmission electron microscopy (TEM; JOEL JEM-1010, Japan). Briefly, after co-incubation with $P$. gingivalis, hGFs were washed with PBS and fixed with $2.5 \%$ glutaraldehyde. They were then scraped off, dehydrated, embedded, and sectioned before TEM observation. ${ }^{25}$

\section{Effects of PSC-SPIONs on P. gingivalis LPS- Induced ROS Production in hGFs}

Cytoplasmic ROS production in hGFs induced by $P$. gingivalis LPS (Invivogen, France) was assessed using the fluorescent probe DCFH-DA (Beyotime, China). ${ }^{28}$ Briefly, hGFs were seeded in a black-walled clear-bottom 96-well plate at $10^{4}$ cells/well. hGFs co-cultured with $1 \mu \mathrm{g} / \mathrm{mL} P$. gingivalis LPS for $24 \mathrm{~h}$ were used as the positive control (hGF+LPS), and untreated hGFs were used as the negative control (hGF). hGFs in the PSC-SPION groups were pretreated with 5, 15, or 25 $\mu \mathrm{g} / \mathrm{mL}$ PSC-SPIONs for $6 \mathrm{~h}$ and then stimulated with 1 $\mu \mathrm{g} / \mathrm{mL}$ LPS for $24 \mathrm{~h}$. The experimental groups were named 5-P-S+LPS, 15-P-S+LPS, and 25-P-S+LPS. The hGFs were washed and incubated in the dark with 
DCFH-DA (1:2000) for $30 \mathrm{~min}$. Fluorescence intensity was measured using a multimode microplate reader (excitation $488 \mathrm{~nm}$, emission $525 \mathrm{~nm}$; SpectraMax $\mathrm{M}^{\mathrm{e}}$, USA). The hGFs were also visualized using inverted fluorescence microscopy (Leica DMI3000B, Germany).

\section{Effect of PSC-SPIONs on P. gingivalis LPS- Induced Inflammatory Response in hGFs}

To investigate the effects of PSC-SPIONs on the inflammatory response in hGFs, we treated hGFs with $1 \mu \mathrm{g} / \mathrm{mL}$ P. gingivalis LPS. The positive control, negative control, and the three experimental groups including 5-P-S+LPS, 15-P-S+LPS, and 25-P-S+LPS were the same as those described in the previous section. The hGFs and culture supernatants were collected for subsequent experiments.

To establish the effects of PSC-SPIONs on LPSinduced inflammatory cytokine production, the levels of IL-1 $\beta$, IL-6, IL- 8 , and TNF- $\alpha$ in the cell culture supernatants were measured using an enzyme-linked immunosorbent assay (ELISA) kit (Neobioscience, China). ${ }^{29}$

The mRNA levels of $I L-1 \beta, I L-6, I L-8, T N F-\alpha, C O X$ 2 , and $T L R-4$ were measured by quantitative reverse transcription-polymerase chain reaction (qRT-PCR) analysis. hGFs were lysed using Trizol reagent (Ambion, USA). Total RNA was extracted using a PureLink ${ }^{\mathrm{TM}}$ RNA Mini Kit (Invitrogen, USA). cDNA was synthesized using PrimeScript ${ }^{\mathrm{TM}}$ RT Master Mix (Takara, Japan). qRT-PCR was performed using gene-specific primers and PowerUp ${ }^{\mathrm{TM}}$ SYBR $^{\mathrm{TM}}$ Green Master Mix (Applied Biosystems, USA). The primers used are shown in Table 1. The PCR conditions were: $2 \mathrm{~min}$ at $50{ }^{\circ} \mathrm{C}, 2$ min at $95{ }^{\circ} \mathrm{C}$, then 40 cycles of $95{ }^{\circ} \mathrm{C}$ for $15 \mathrm{~s}$ and $60{ }^{\circ} \mathrm{C}$ for $1 \mathrm{~min}$. The relative gene expression for each sample was normalized to that of GAPDH. Data were analyzed using the $2^{-\mathrm{ddCt}}$ method $(\mathrm{n}=3)$.

\section{Effects of PSC-SPIONs on the NF-kB-P65 Pathway}

Cells from the 15-P-S group stimulated by LPS for $1 \mathrm{~h}$ (15-P-S+LPS) were used for this study. The hGFs were digested with trypsin for 2 min and centrifuged at $500 \times \mathrm{g}$ for $5 \mathrm{~min}$. NE-PER ${ }^{\mathrm{TM}}$ nuclear and cytoplasmic extraction reagents (Thermo Fisher, USA) were used to isolate the cytoplasmic and nuclear proteins, respectively. Protein concentrations were measured with enhanced BCA Protein Assay Kits (Beyotime, China). Protein samples were mixed with $5 \times$ sodium dodecyl sulfate-polyacrylamide gel electrophoresis (SDS-PAGE) sample loading buffer (Beyotime, China). The protein mixtures were denatured at $100{ }^{\circ} \mathrm{C}$ for $5 \mathrm{~min}$. Samples were loaded onto $10 \%$ SDS-PAGE (Beyotime, China) at $20 \mu \mathrm{g}$ protein/lane and transferred to polyvinylidene fluoride membranes after resolution. Membranes were blocked in 5\% non-fat milk for $2 \mathrm{~h}$ and incubated with primary antibody (1:1000) overnight at $4{ }^{\circ} \mathrm{C}$ and then incubated with secondary antibody (1:5000) for $1 \mathrm{~h}$. The loading controls were GAPDH (in the cytoplasmic fraction) and Histone 3 (H3) (in the nuclear fraction). The primary antibodies used were: rabbit anti-GAPDH (Proteintech, USA), rabbit antiH3 (Proteintech), rabbit anti-NF-кB-p65 (Cell Signaling Technology, CST, USA), rabbit anti-phospho-NF-кB-p65 (CST), mouse anti-IкB $\alpha$ (CST), and mouse anti-phospho$\mathrm{I} \kappa \mathrm{B} \alpha$ (CST, USA). The secondary antibodies used were: Peroxidase-conjugated AffiniPure Goat Anti-mouse IgG $(\mathrm{H}+\mathrm{L})$ and Peroxidase-conjugated AffiniPure Goat Antirabbit $\operatorname{IgG}(\mathrm{H}+\mathrm{L})$ (both from Proteintech).

\section{Statistical Analysis}

Data are presented as the mean \pm standard deviation (SD) of three independent experiments. Statistical analysis was performed using GraphPad Prism software v7.04. Group comparisons between two samples were performed by $t$-test, while multiple comparisons among three or more

Table I Primer Sequences Used for hGF Gene Expression Analysis

\begin{tabular}{|l|l|l|}
\hline Gene (Human) & Forward $\mathbf{( 5}^{\prime} \mathbf{- 3} \mathbf{3}^{\prime} \mathbf{)}$ & Reverse $\mathbf{( 5}^{\prime} \mathbf{- 3} \mathbf{)}$ \\
\hline GAPDH & ACCACAGTCCATGCCATCAC & ACCACCCTGTTGCTGTA \\
IL-6 & ATAACCACCCCTGACCCAAC & CCCATGCTACATTTGCCGAA \\
IL-8 & TCAGAGACAGCAGAGCACAC & GGCAAAACTGCACCTTCACA \\
IL-I $\beta$ & TAGGGCTGGCAGAAAGGGAACA & GTGGGAGCGAATGACAGAGGGT \\
TNF- $\alpha$ & AACATCCAACCTTCCCAAACGC & TGGTCTCCAGATTCCAGATGTCAGG \\
COX-2 & CTGGAACATGGAATTACCCAGTTTG & TGGAACATTCCTACCACCAGCA \\
TLR-4 & CACAGACTTGCGGGTTCTACATC & GGACTTCTAAACCAGCCAGACCTTG \\
\hline
\end{tabular}


groups were conducted using one-way analysis of variance with Dunnett's test. Differences were considered significant at $P<0.05$.

\section{Results}

\section{PSC-SPIONs are Biocompatible with} hGFs and Have a Negligible Inhibitory Effect on $P$. gingivalis

hGFs were identified by fluorescence staining; they are negative for keratin and positive for vimentin (Figure S1). Based on the results of CCK8 assay, no appreciable cytotoxic effects on hGFs were observed for PSC-SPIONs in the concentration range 5 to $100 \mu \mathrm{g} / \mathrm{mL}$ following treatment for up to $72 \mathrm{~h}$ (Figure 1A). These data suggest that PSCSPIONs at up to $100 \mu \mathrm{g} / \mathrm{mL}$ are biocompatible with hGFs. In a preliminary study, we found that high concentrations of PSC-SPIONs (50 and $100 \mu \mathrm{g} / \mathrm{mL}$ ) did not inhibit the expression of proinflammatory cytokines in hGFs stimulated with $P$. gingivalis LPS (Figure S2). Therefore, PSC-SPION concentrations of 5,15 , and $25 \mu \mathrm{g} / \mathrm{mL}$ were chosen for the subsequent cell assays.

The toxicity of $25 \mu \mathrm{g} / \mathrm{mL}$ PSC-SPIONs toward $P$. gingivalis was determined by colony counting. No significant difference in colony number between the P-S-treated $P . g$ and $P$. $g$ control groups was observed after treatment for 2 $\mathrm{h}$ (Figure 1B). SEM analysis revealed that PSC-SPIONs do not affect the morphology of $P$. gingivalis (Figure 1C and D). These results indicate that PSC-SPIONs at $25 \mu \mathrm{g} /$ $\mathrm{mL}$ have a negligible inhibitory effect on $P$. gingivalis.

\section{PSC-SPIONs Prevent Invasion of hGFs by}

\section{$P$. gingivalis}

The effect of PSC-SPIONs on the infiltration of hGFs by $P$. gingivalis was studied. There was no significant reduction in the invading $P$. gingivalis colony number in the 5$\mathrm{P}-\mathrm{S}+P . g$ group compared with the $\mathrm{hGF}+P . g$ control, and there was a minor but not statistically significant reduction
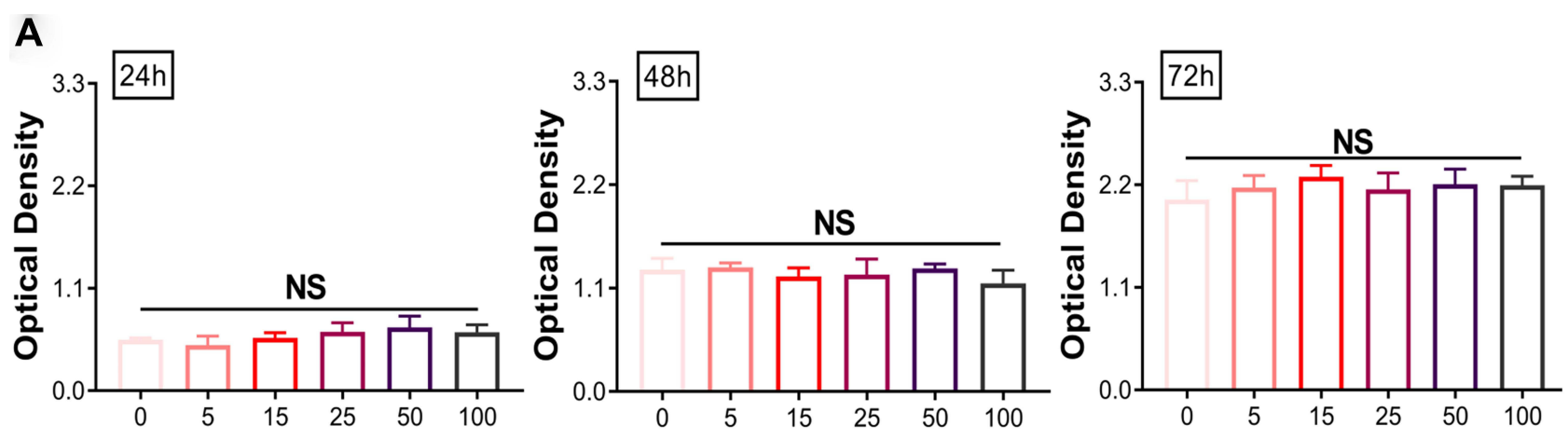

Concentration of P-S $(\mu \mathrm{g} / \mathrm{mL})$

B

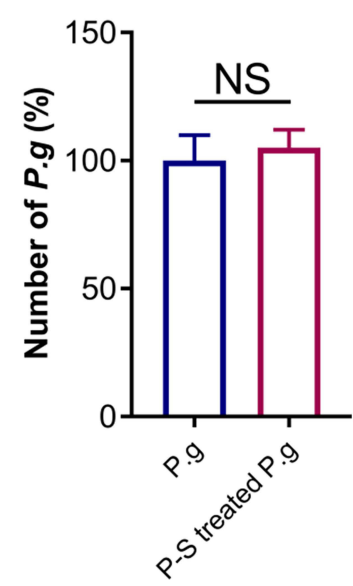

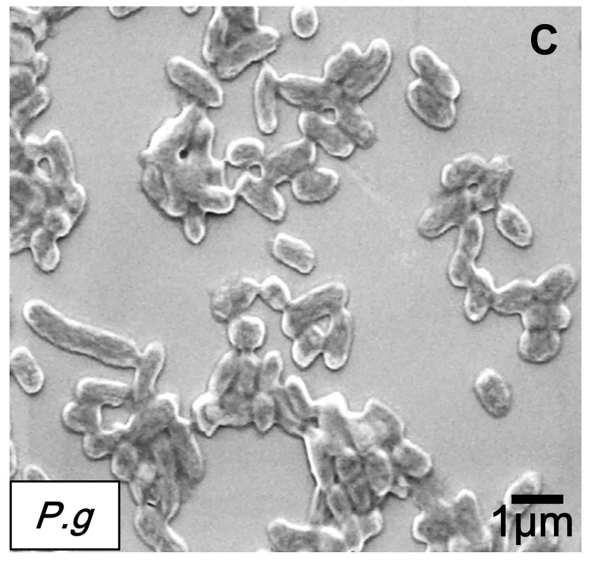

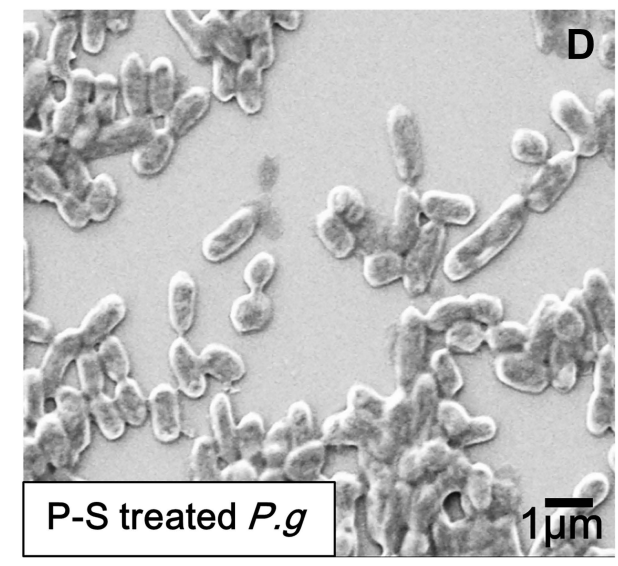

Figure I Effect of polyglucose sorbitol carboxymethyl ether-superparamagnetic iron oxide nanoparticles (PSC-SPIONs) on the viability of human gingival fibroblasts (hGFs) and $P$. gingivalis $(n=5)$. (A) hGF viability was determined using Cell Counting Kit-8 assays after treatment with different concentrations of PSC-SPIONs for 24,48 , and $72 \mathrm{~h}$. Data are presented as the mean optical density \pm SD. (B) $P$. gingivalis colony numbers (\%) after $25 \mu g / \mathrm{mL}$ PSC-SPION treatment for $2 \mathrm{~h}$ with untreated $P$. gingivalis as the control. (C and D) Morphology of $P$. gingivalis after $25 \mu \mathrm{g} / \mathrm{mL}$ PSC-SPIONs treatment for $2 \mathrm{~h}$ (D) with untreated $P$. gingivalis as the control (C). Abbreviations: P-S, PSC-SPIONs; NS, not significant. 
for the 25-P-S $+P . g$ group. However, there was a significant decrease for the 15-P-S $+P . g$ group $(P<0.01$; Figure 2A). Consistently, CLSM images show that the number of SYTO-9-labeled green-fluorescent $P$. gingivalis in hGFs was much lower in the $15-\mathrm{P}-\mathrm{S}+P$. $g$ group than in the hGF $+P . g$ control (Figure 2B). TEM images show that $P$. gingivalis adheres to the hGF cell membrane and induces clear invagination and ruffles (Figure 2C), which is a precursor of endocytosis. ${ }^{30}$ This change was barely observed in the 15-P-S $+P$. $g$ group, but structures resembling endocytic vesicles (yellow arrows) were observed. These data suggest that PSC-SPIONs prevent the entry of $P$. gingivalis into hGFs.

To test the internalization of PSC-SPIONs by hGFs, inductively coupled plasma-optical emission spectrometry was used to determine the iron content of hGFs after $6 \mathrm{~h}$ of co-culture with PSC-SPIONs. The iron contents for the 15-P-S and 25-P-S groups were higher than that for the hGF control group (Figure S3), while that for the 5-P-S group was as low as that of the hGF control group. These results indicate that the inhibition of hGF invasion by $P$. gingivalis is related to the internalization of PSC-SPIONs.

\section{PSC-SPION Treatment Decreases ROS Levels in hGFs Induced with $P$. gingivalis LPS}

As has been reported previously, ${ }^{31} P$. gingivalis-LPS treatment markedly increased ROS levels in hGFs $(P<0.01)$, which presents as more intense and denser green fluorescence in the cells of the hGF+LPS group in Figure 3. However, the density of green fluorescence was clearly decreased in the 5-P-S+LPS, 15-P-S+LPS, and 25-P-S +LPS groups compared with that in hGF+LPS group (Figure 3). The quantitative data match the qualitative results, revealing a similar decrease in ROS levels in all the experimental groups compared with the hGF+LPS positive control $(P<0.01)$. Furthermore, the extent of reduction was similar for all the experimental groups. These results show that PSC-SPIONs decrease the $P$. gingivalis LPS-induced ROS level in hGFs.

\section{PSC-SPIONs Decrease P. gingivalis LPS- Induced Inflammatory Response in hGFs}

Inflammatory cytokine levels in cell supernatants were determined by ELISA (Figure 4A). Significantly increased inflammatory cytokine secretion was detected in hGF+LPS group compared with the hGF negative control and three
P-S+LPS groups $(P<0.01)$. The 5-P-S+LPS, 15-P-S + LPS, and 25-P-S+LPS groups all showed significantly lower LPS-induced IL-1 $\beta$, TNF- $\alpha$, IL-6, and IL-8 levels than the hGF+LPS positive control group $(P<0.05)$. The 15-P-S+LPS group showed greater inhibition of IL-1 $\beta$, TNF- $\alpha$, and IL-6 than the 5-P-S+LPS and 25-P-S+LPS groups $(P<0.05$; Figure 4A).

qRT-PCR was used to quantify the expression of these markers at the mRNA level (Figure 4B). As expected, the expression of $I L-1 \beta, T N F-\alpha, I L-6, I L-8$, and $C O X-2$ was significantly elevated in the hGF+LPS positive control group compared with the hGF negative control and three P-S+LPS groups $(P<0.01)$. Comparatively, the 5-P-S +LPS, 15-P-S+LPS, and 25-P-S+LPS groups showed downregulation of these proinflammatory cytokines $(P<$ $0.01)$. The 15-P-S+LPS group showed the highest downregulation of $I L-1 \beta, T N F-\alpha$, and $I L-8(P<0.05$; Figure 4B). Therefore, PSC-SPION treatment decreases the $P$. gingivalis LPS-induced inflammatory response of hGFs, and $15 \mu \mathrm{g} /$ $\mathrm{mL}$ is the optimal PSC-SPION concentration. In addition, the expression of TLR-4 was inhibited by treatment with PSC-SPIONs, indicating that the NF- $\kappa B$ signaling pathway is involved in their mechanism of action. ${ }^{14}$

\section{PSC-SPIONs Inhibit the $P$. gingivalis LPS- Induced Activation of the NF- $\kappa$ B Signaling Pathway}

To elucidate the anti-inflammatory mechanism of PSCSPIONs, Western blot analysis was used to determine levels of $\mathrm{p} 65, \mathrm{P}$-p65, I $\mathrm{KB} \alpha$, and P-І $\kappa \mathrm{B} \alpha$ proteins (Figure 5). P. gingivalis-LPS stimulation leads to the phosphorylation and degradation of I $\mathrm{I} B \alpha$ in the cell lysates. ${ }^{26}$ Consistently, IкB $\alpha$ level was decreased $(P<0.05)$ and P-IкB $\alpha(P<0.01)$ and P-p65 levels were increased $(P<$ 0.01 ) in hGF+LPS group. However, the 15-P-S+LPS group showed inhibition of NF- $\kappa \mathrm{B}$ pathway activation via the downregulation of P-IкB $\alpha$ and P-p65 proteins ( $P$ $<0.01)$. The degradation of IкB $\alpha$ was also inhibited in the 15-P-S+LPS group $(P<0.05$; Figure 5A).

Another important event in the activation of NF- $\mathrm{KB}$ signaling is the translocation of $\mathrm{p} 65$ from the cytoplasm to the nucleus. ${ }^{26}$ Therefore, nuclear p65 protein levels were also determined. $P$. gingivalis LPS-induced upregulation of nuclear p65 (N-p65) was inhibited in the 15-P-S group $(P<$ 0.01 ; Figure 5B). These findings indicate that PSC-SPIONs inhibit $P$. gingivalis LPS-induced inflammation in hGFs by downregulating NF- $\mathrm{kB}$ signaling pathway proteins. 

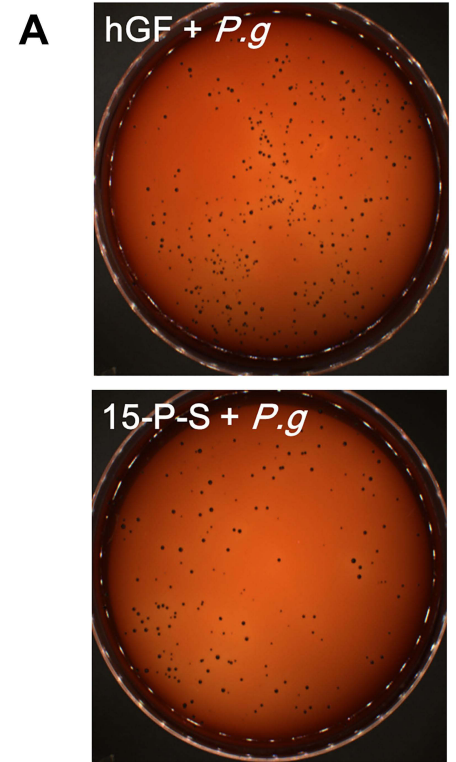

B
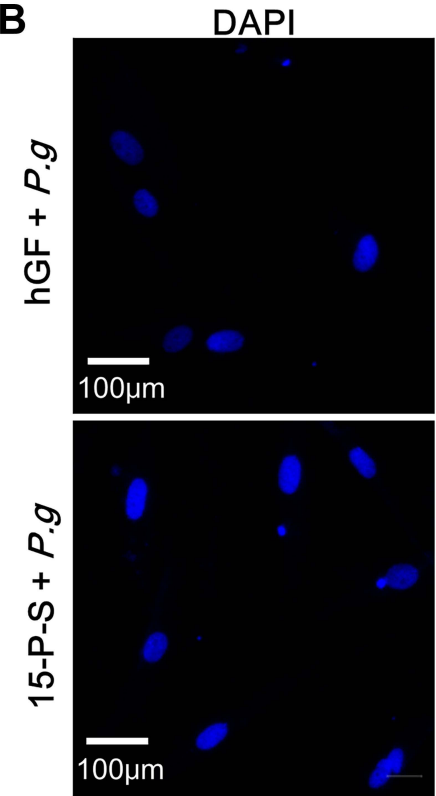

C

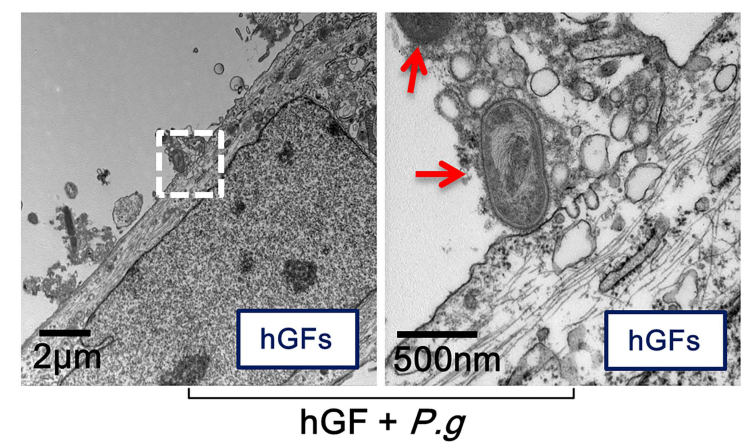

Phalloidin
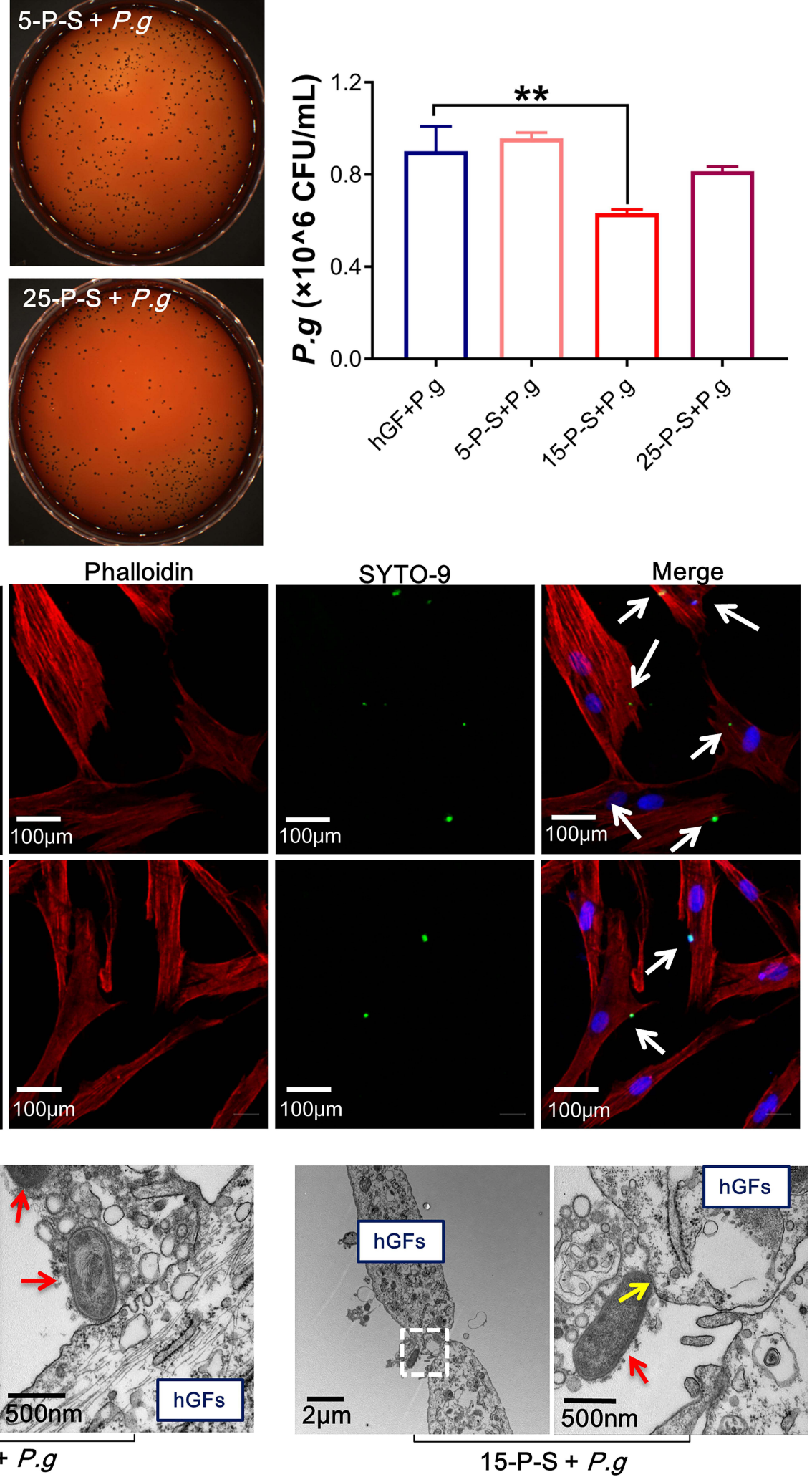

Figure 2 Anti-hGF-invasion effect of PSC-SPIONs on P. gingivalis. (A) Colony numbers of invading P. gingivalis in P-S treated hGFs ( $\mathrm{n}=3$ ). $(\mathbf{B})$ Confocal laser scanning microscopy images of I5-P-S-treated hGFs invaded by P. gingivalis (white arrows). Cell skeletal protein was stained with Alexa Fluor ${ }^{\circledR} 594$ Phalloidin (red fluorescence). Nuclei were stained with DAPI (blue fluorescence). P. gingivalis were stained with SYTO-9 (green fluorescence). (C) Transmission electron microscopy (TEM) images of I5-P-S-treated hGFs invaded by $P$. gingivalis (red arrows). Structures resembling endocytic vesicles are marked by yellow arrows. $* * P<0.01$ compared with hGF+P. $g$ control group. Abbreviation: P-S, PSC-SPIONs. 

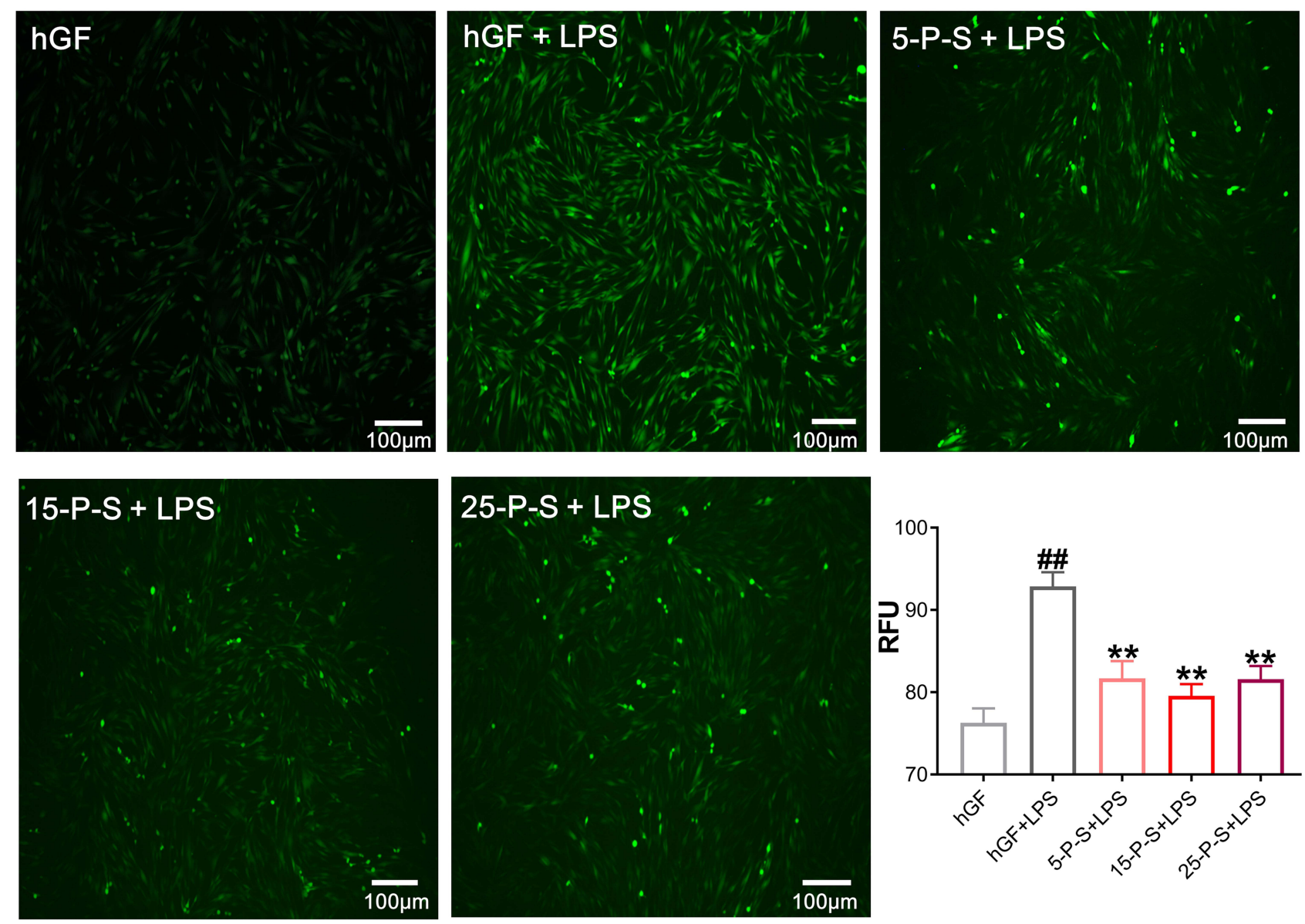

Figure 3 Effect of PSC-SPIONs on P. gingivalis lipopolysaccharide (LPS)-induced reactive oxygen species (ROS) production in hGFs. Inverted fluorescence microscopy images show cytoplasmic ROS in P-S treated hGFs stimulated by P. gingivalis LPS. Quantitative measurement of cytoplasmic ROS in P-S-treated hGFs stimulated by P. gingivalis LPS was performed using a multimode microplate reader. Data are presented as the mean $\pm S D(n=3)$. ${ }^{\prime} P<0.01$ compared with hGF negative control; $* * P<0.01$ compared with hGF+LPS positive control.

Abbreviations: P-S, PSC-SPIONs; RFU, relative fluorescence units.

\section{The Anti-Inflammatory Effect of PSC- SPIONs is Mainly Due to Their} Nanoscale Size, Not the Coating Material Both the nanosize effect of PSC-SPIONs which has direct impacts on their surface area and interfacial properties and/or their PSC coating material could be responsible for their anti-inflammatory activity. Therefore, SPIONs with a similar iron core to that of PSC-SPIONs but surface-modified with dimercaptosuccinic acid (DMSASPIONs, abbreviated to D-S in the group labels) instead of PSC were obtained (Nanjing Nanoeast Biotech Co., Ltd.). PSC that was not conjugated to iron cores was also obtained (Jiangsu Key Laboratory for Biomaterials and Devices). The effects of these two materials on $P$. gingivalis LPS-induced inflammation in hGFs were assessed.
First, PSC was used to treat $P$. gingivalis LPS-induced inflammation. The experimental groups were named 5PSC+LPS, 15-PSC+LPS, and 25-PSC+LPS. In each group, the amount of PSC was corresponded to that in the 5-P-S, 15-P-S, and 25-P-S groups, respectively. Untreated hGFs were used as the negative control, and LPS-stimulated hGFs were used as the positive control (hGF+LPS). Compared with the hGF+LPS positive control, the 5-PSC+LPS, 15-PSC+LPS, and 25-PSC+LPS groups all showed suppression of $I L-1 \beta$ upregulation $(P$ $<0.05$ ), and the 5-PSC+LPS and 15-PSC+LPS groups showed suppression of $C O X-2$ upregulation $(P<0.01$; Figure 6). However, PSC treatment did not inhibit the upregulation of $T N F-\alpha, I L-6$, or $I L-8$. Furthermore, increased $C O X-2$ expression was observed for the 25PSC+LPS group $(P<0.01$; Figure 6$)$. 


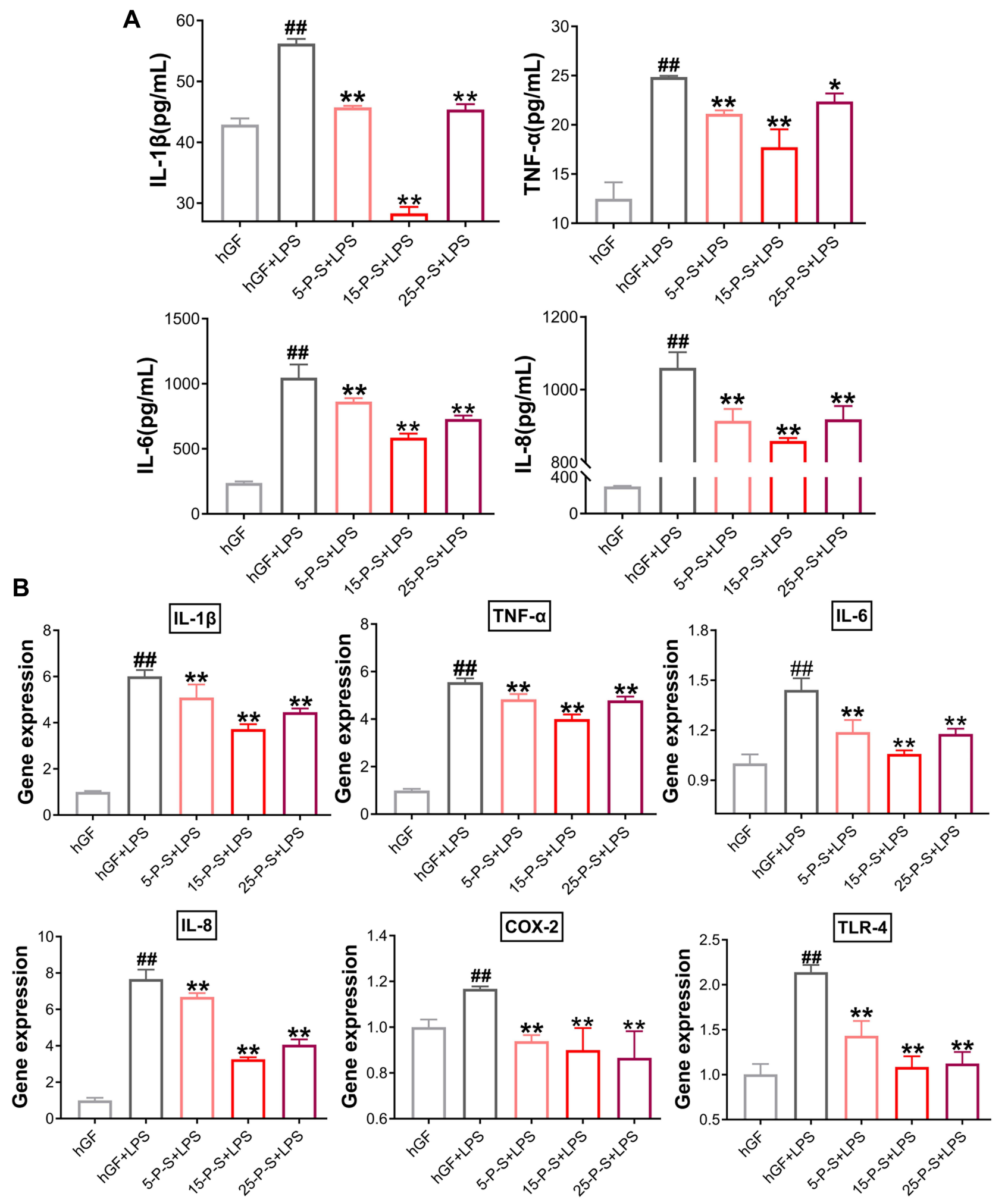

Figure 4 Effects of PSC-SPIONs on $P$. gingivalis LPS-induced inflammatory response in hGFs (A). IL-I $\beta$, TNF- $\alpha$, IL-6, and IL-8 levels in P-S-treated hGFs stimulated by $P$. gingivalis LPS measured using enzyme-linked immunosorbent assays. (B) Gene expression (mRNA levels) of IL-I $\beta$, TNF- $\alpha$, IL-6, IL-8, COX-2, and TLR-4 in P-S-treated hGFs stimulated by $P$. gingivalis LPS. Histograms show means \pm SD from three independent experiments. ${ }^{\#} P<0.01$ compared with hGF negative control group; ${ }^{*} P<0.05$, $* * P<$ 0.01 compared with hGF+LPS positive control group.

Abbreviation: P-S, PSC-SPIONs. 
A
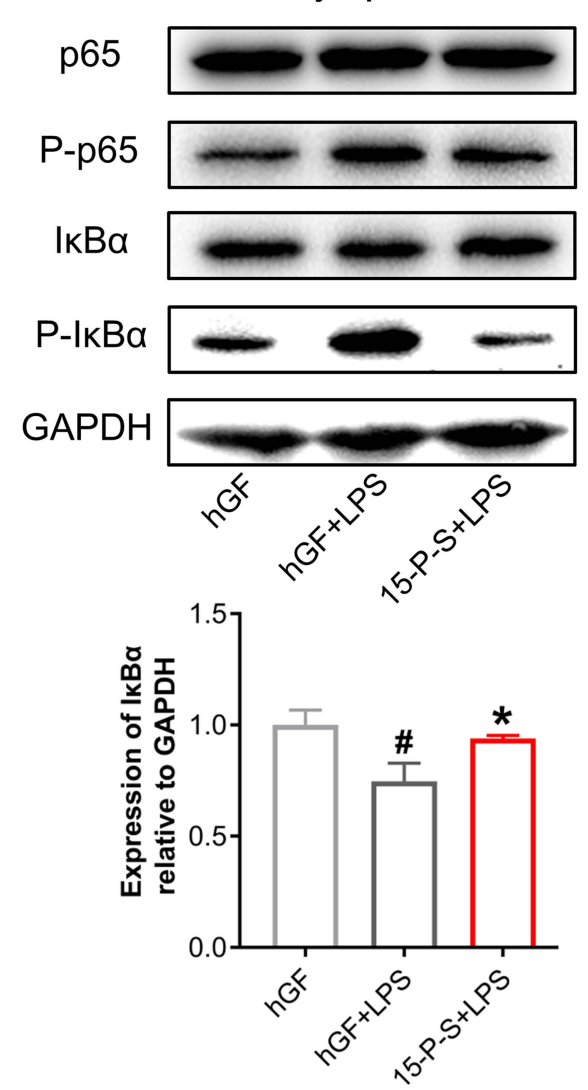
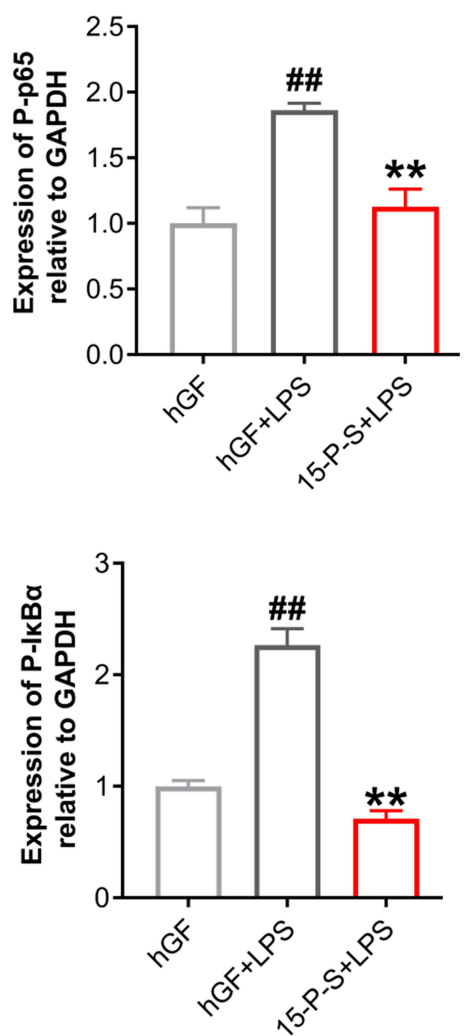

B
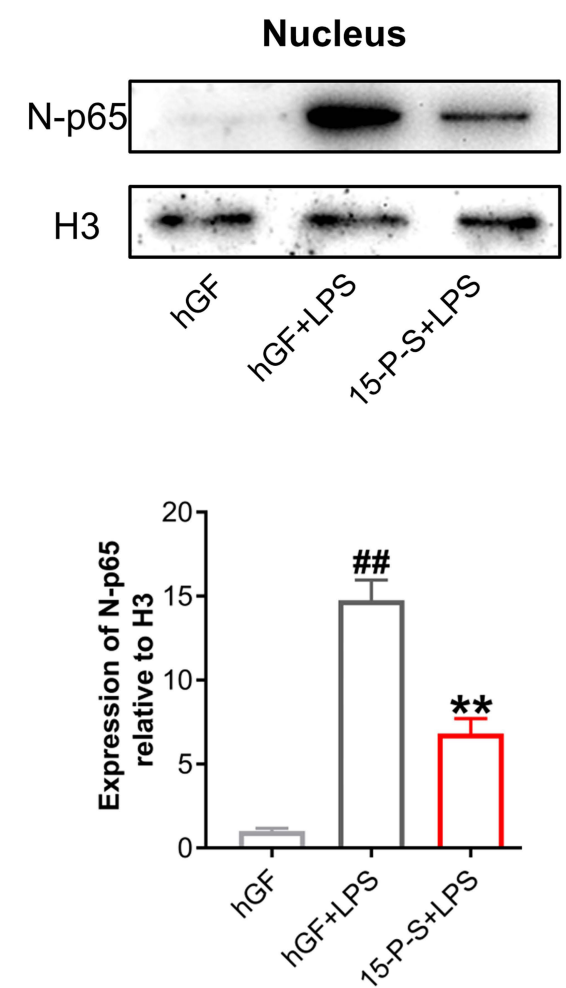

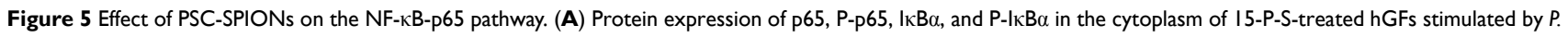
gingivalis LPS. (B) Protein expression of $\mathrm{p} 65$ in the nuclei of I5-P-S-treated hGFs stimulated by $P$. gingivalis LPS. Histograms show means \pm SD from three independent experiments. ${ }^{\#} P<0.05,{ }^{\# \#} P<0.01$ compared with hGF negative control; ${ }^{*} P<0.05,{ }^{* *} P<0.01$ compared with hGF+LPS positive control.

Abbreviation: P-S, PSC-SPIONs.

Second, PSC-SPIONs were analyzed using a SQUID magnetometer to confirm their magnetic properties. From the magnetic hysteresis loops of PSC-SPIONs, they exhibited superparamagnetic characteristics with saturation magnetization values of $73 \mathrm{emu} / \mathrm{g}$. Remnant magnetization was not observed when the magnetic field was removed (Figure 7A).

Then, DMSA-SPIONs (abbreviated to D-S in the group labels) were used to treat $P$. gingivalis LPS-induced inflammation. The morphology of PSC-SPIONs and DMSA-SPIONs is similar-both are spherical particles with diameter $<20 \mathrm{~nm}$ (Figure 7B). Their major parameters are shown in Table 2. Like PSC-SPIONs, DMSASPIONs also have $\gamma-\mathrm{Fe}_{2} \mathrm{O}_{3}$ cores and carboxyl groups on the surface, leading to a negative surface charge. The concentrations of DMSA-SPIONs tested were also $5 \mu \mathrm{g} /$ $\mathrm{mL}, 15 \mu \mathrm{g} / \mathrm{mL}$, and $25 \mu \mathrm{g} / \mathrm{mL}$. Therefore, the experimental groups are named 5-D-S+LPS, 15-D-S+LPS, and 25-DS+LPS. The negative and positive control groups were untreated hGFs and $P$. gingivalis LPS-stimulated hGFs
(hGF+LPS), respectively. Compared with the positive control, the 5-D-S+LPS, 15-D-S+LPS, and 25-D-S+LPS groups showed significant suppression of $I L-1 \beta, T N F-\alpha$, $I L-6, I L-8$, and $C O X-2$ upregulation $(P<0.01$; Figure $7 \mathrm{C})$.

These results indicate that the anti-inflammatory activity of PSC-SPIONs is due to their nanosize effect, which prevents the internalization of pathogenic bacteria and the binding of LPS to TLR-4, rather than the surface coating material of the nanoparticles.

\section{Discussion}

Inflammation caused by $P$. gingivalis invasion and stimulation by its LPS in hGFs plays an important role in the development of periodontal diseases. In this study, we demonstrate that PSC-SPIONs prevent $P$. gingivalis invasion and inhibit inflammatory responses to $P$. gingivalis LPS, including the generation of ROS and the expression of inflammatory mediators, by suppressing TLR-4 and NF$\kappa \mathrm{B}$ signaling. 


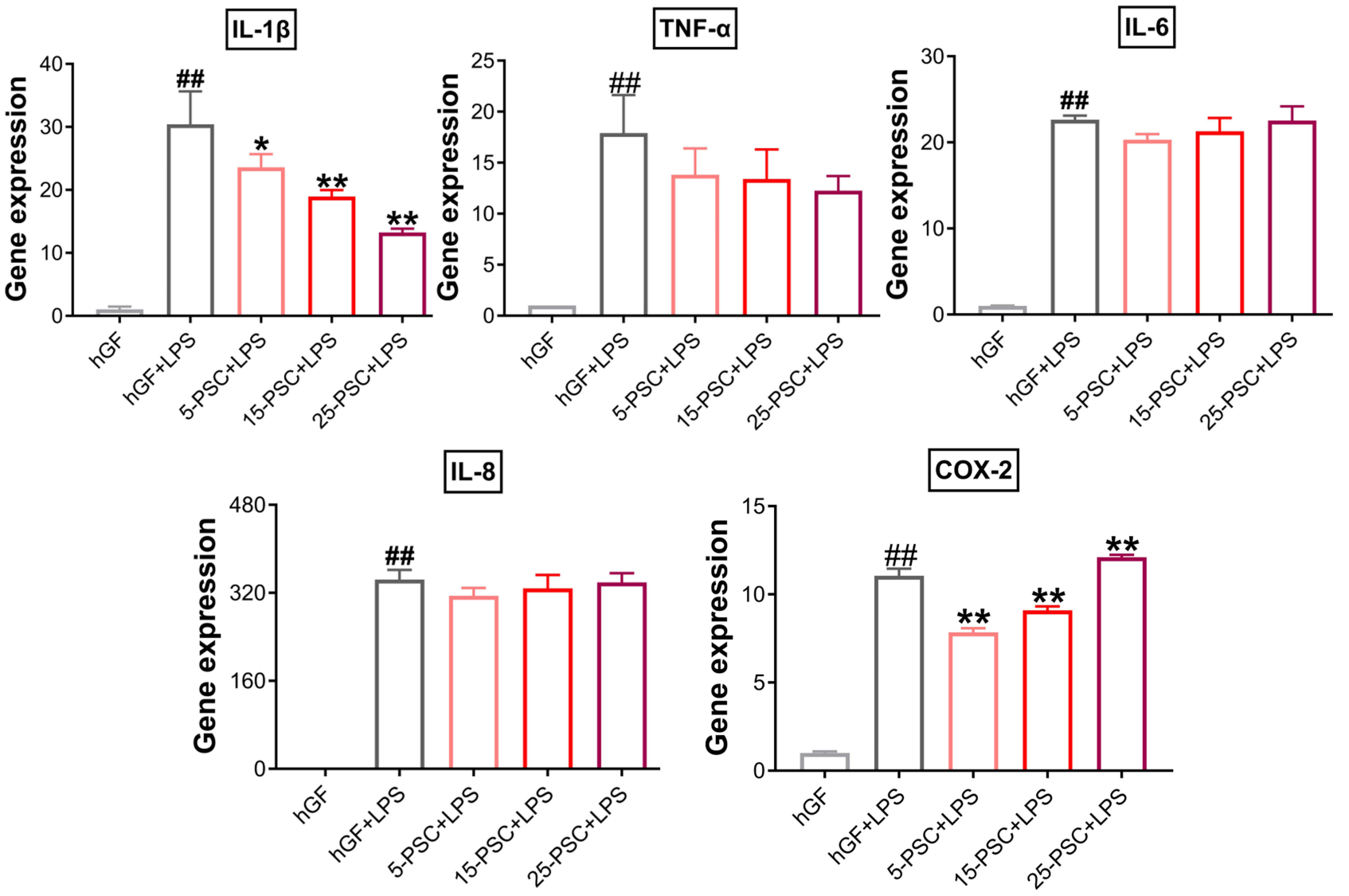

Figure 6 Effect of PSC on P. gingivalis LPS-induced inflammatory response in hGFs. Gene expression (mRNA levels) of IL-I $\beta$, TNF- $\alpha$, IL-6, IL-8, and COX-2 were quantified in PSC-treated hGFs stimulated with $P$. gingivalis LPS using quantitative reverse transcription-polymerase chain reaction. Histograms show means \pm SD from three independent experiments. ${ }^{\#} P<0.01$ compared with hGF negative control; $* P<0.05$, ${ }^{* * P}<0.01$ compared with hGF+LPS positive control.

Biosafety is the most important consideration for the medical application of a material. DMAS-SPIONs appear to be effective in alleviating $P$. gingivalis LPS-induced inflammation (Figure 7). However, the DMSA coating can negatively affect cellular gene expression. ${ }^{32}$ In contrast, PSC is a polysaccharide that has been widely applied in clinical treatment. Polysaccharides have been shown to scavenge ROS in vitro. ${ }^{33}$ Therefore, PSC-SPIONs are preferable in the context of biosafety. They are nontoxic to BMSCs at concentrations as high as $500 \mu \mathrm{g} / \mathrm{mL} .{ }^{20}$ In this study, they showed good cytocompatibility with hGFs at $100 \mu \mathrm{g} / \mathrm{mL}$ (Figure 1A).

Moreover, the PSC-SPIONs prepared by our methods have better properties such as more uniform particle size distribution, more regular morphology, and better magnetism and crystallinity than the nano drug Ferumoxytol (Feraheme ${ }^{\mathrm{TM}}$ ). ${ }^{27,34}$ Our PSC-SPIONs have been well characterized by TEM, particle size analysis, Fouriertransform infrared spectrometry, X-ray diffraction, vibrating sample magnetometry, thermogravimetric analysis, and heating measurement in an alternating-current magnetic field. ${ }^{27,34}$ Here, we retested the superparamagnetic characteristics (Figure 7A), morphology (Figure 7B), and hydrodynamic size (Table 2) of the PSC-SPIONs. The results were all consistent with previous reports. ${ }^{27,34}$

PSC-SPIONs are recognized by scavenger receptor A (SR-A) and introduced to cells by endocytosis through clathrin-dependent pathways, with or without transfection protocols using protamine sulfate and heparin. ${ }^{35}$ Therefore, the iron content of hGFs incubated with PSCSPIONs was obviously increased (Figure S3). According to a previous study, the iron content of rat adipose-derived stem cells was $0.31 \pm 0.02 \mathrm{pg} / \mathrm{cell} .{ }^{36}$ This is consistent with our results, wherein the iron content of untreated hGFs was very low (Figure S3).

The decreased invasion of hGFs by $P$. gingivalis may be related to the internalization of PSC-SPIONs. $P$. gingivalis and PSC-SPIONs are internalized by hGFs through clathrin-based endocytosis via similar 
A

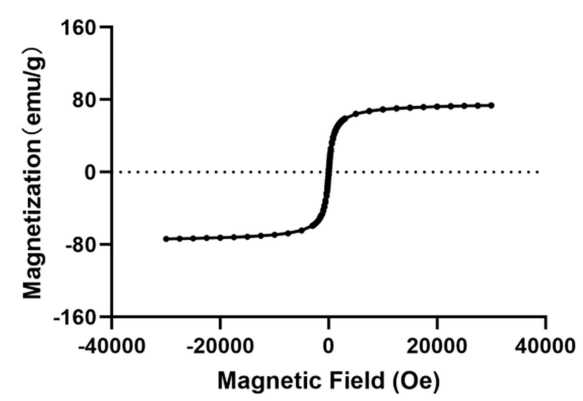

B

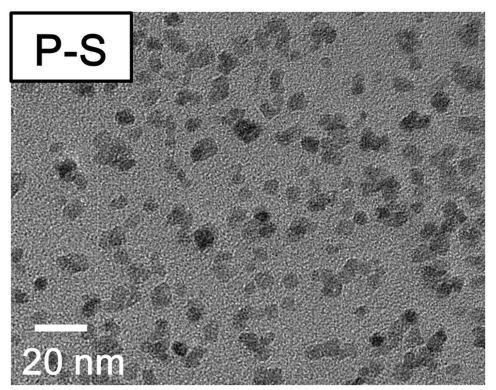

C
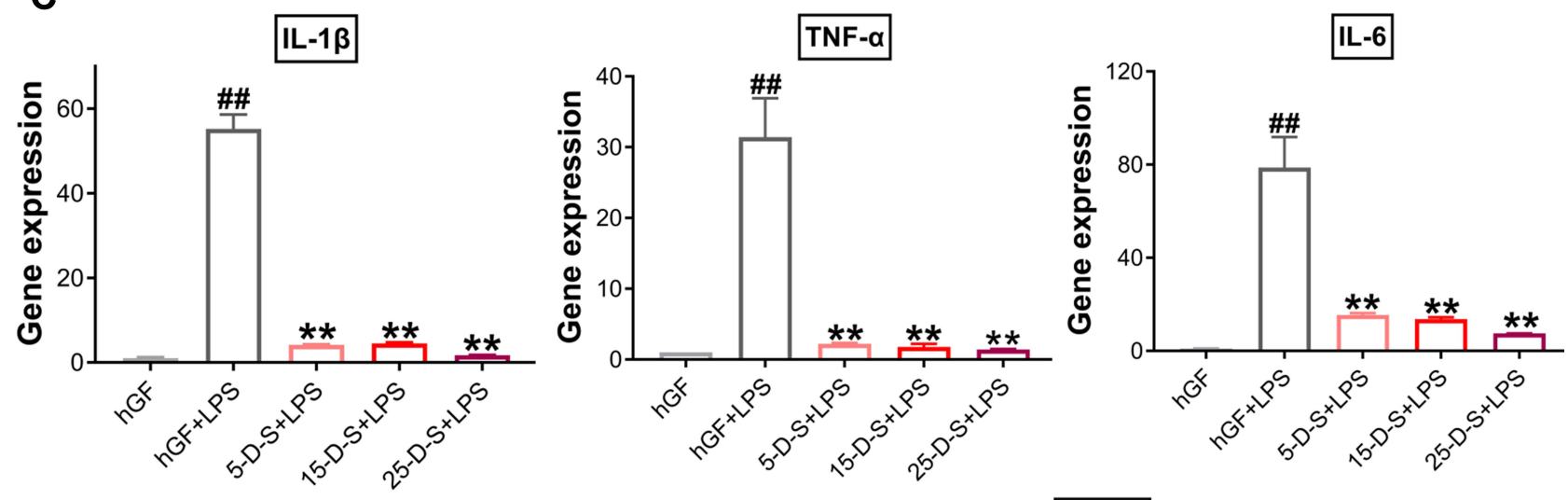

IL-8

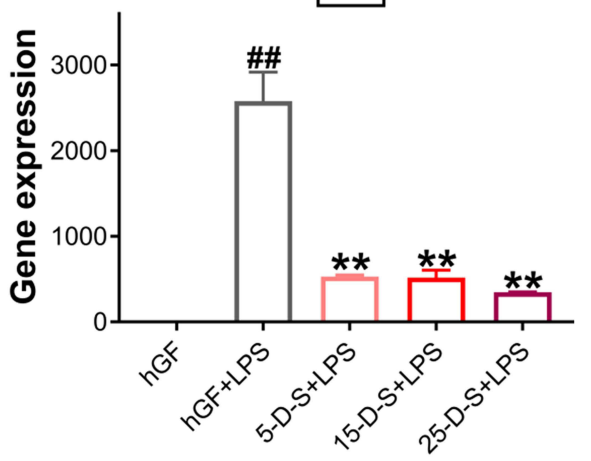

COX-2

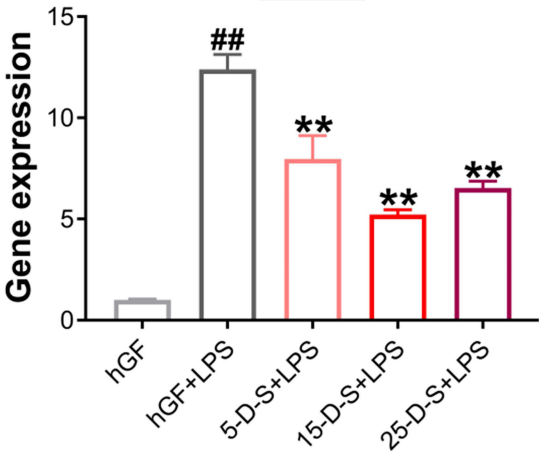

Figure 7 Effect of dimercaptosuccinic acid (DMSA)-SPIONs on P. gingivalis LPS-induced inflammatory response in hGFs. (A) Superconducting quantum interference device (SQUID) magnetometer magnetic hysteresis loops of PSC-SPIONs at $300 \mathrm{~K}$. (B) TEM images of PSC-SPIONs and DMSA-SPIONs. (C) Gene expression (mRNA levels) of IL$I \beta, T N F-\alpha, I L-6, I L-8$, and COX-2 in D-S-treated hGFs stimulated with $P$. gingivalis LPS. Histograms show means \pm SD from three independent experiments. ${ }^{\prime \prime} P<0.0 I$ compared with hGF negative control; $* * P<0.01$ compared with hGF+LPS positive control.

Abbreviations: P-S, PSC-SPIONs; D-S, DMSA-SPIONs.

intracellular trafficking pathways. Boisvert et al suggested that $P$. gingivalis invasion depends on clathrinmediated endocytosis, ${ }^{37}$ while Ho et al demonstrated that clathrin-mediated endocytosis is responsible for the invasion of oral epithelial cells by $P$. gingivalis and its outer membrane vesicles. ${ }^{13}$ So, it is possible

Table 2 Comparison of the Main Structural Parameters of PSC-SPIONs and DMSA-SPIONs

\begin{tabular}{|l|l|l|l|l|l|}
\hline Product & Iron Core & Surface Charge & Surface Group & External Material & Hydrodynamic Size (nm) \\
\hline PSC-SPIONs & $\gamma-\mathrm{Fe}_{2} \mathrm{O}_{3}$ & Negative & Carboxyl & PSC & 34 \\
\hline DMSA-SPIONs & $\gamma-\mathrm{Fe}_{2} \mathrm{O}_{3}$ & Negative & Carboxyl & DMSA & 25 \\
\hline
\end{tabular}


that clathrin-based endocytosis of hGFs is blocked by PSC-SPIONs, preventing the uptake of $P$. gingivalis (Figure 2A). The concentration of PSC-SPIONs in the 5-P-S group may be too low to completely bind clathrin. The 25-P-S group also showed decreased P. gingivalis invasion; however, the results were not statistically significant, possibly because of experimental error.

PSC-SPION pretreatment also suppressed the upregulation of $P$. gingivalis LPS induced ROS level in hGFs which plays important roles in proinflammatory response. ${ }^{38,39}$ The ability to induce or suppress ROS production is the most important effect of nanoparticles in cells. Internalized nanoparticles usually increase intracellular ROS levels; however, they may also be decreased by nanoparticles. For instance, PEG-phospholipid-coated SPIONs have been shown to decrease ROS-induced retinoic acid-neuronal differentiation in mouse embryonic stem cells. ${ }^{40}$ Conversely, intracellular ROS levels in human umbilical vein endothelial cells were increased by decomposition of $\mathrm{H}_{2} \mathrm{O}_{2}$ into hydroxyl radicals upon incubation with PSC-SPIONs. ${ }^{41}$ PSC-SPIONs decrease ROS levels in myeloid-derived suppressor cells, decreasing late-sepsis immunosuppression. ${ }^{42}$ Moreover, PSC-SPIONs have been shown to scavenge excess ROS by activating the cellular Nrf2-HO-1 pathway to promote osteogenesis and inhibit osteoclast differentiation in iron accumulation-related osteoporosis. ${ }^{23}$ Thus, PSC-SPIONs can increase ROS levels, but may scavenge ROS in diseased conditions, such as those associated with LPS-mediated inflammation.

The protective effects of PSC-SPIONs also presented via decreased proinflammatory cytokine expression in hGFs treated with $P$. gingivalis LPS. Previous studies have shown that $P$. gingivalis LPS activates TLR-4 and its downstream pathways such as the NF- $\kappa B$ pathway, which is critical in the production of cytokines, chemokines, and adhesion molecules. ${ }^{43}$ All the three concentrations of PSC-SPIONs used in this study effectively downregulated inflammatory mediators, but not in a dose-dependent manner (Figure 4). The anti-inflammatory effect increased from 5 to $15 \mu \mathrm{g} / \mathrm{mL}$ PSC-SPIONs and then deceased from 15 to $25 \mu \mathrm{g} / \mathrm{mL}$ PSCSPIONs, likely because higher concentrations of PSCSPIONs can induce inflammation (Figure S2).

Whether SPIONs activate or inhibit TLR-4 depends on the conditions. As with ROS production, SPIONs alone activate TLR-4, but they inhibit it under LPS stimulation. Jin et al reported that Resovist and Feraheme promote macrophage autophagy and inflammatory response by activation of
TLR-4 signaling. ${ }^{44}$ Mulens-Arias et al reported that polyethylenimine-coated SPIONs trigger macrophage activation through TLR-4 signaling and ROS production. ${ }^{45}$ Grosse et al reported that SPIONs coated with a monolayer of oleic acid and a monolayer of amphiphilic polymer suppress LPSinduced NF- $\mathrm{kB}$ activation and production of proinflammatory cytokines in primary human monocytes. ${ }^{25}$ These effects corresponded with impaired LPS internalization by monocytes in the presence of SPIONs, caused by the adsorption of LPS onto the nanoparticle surfaces. ${ }^{25}$ Consistent with our results, Chen et al reported that PEG-coated SPIONs inhibited LPS-induced production of IL- 6 and TNF- $\alpha$ by downregulating the expression of TLR-4, while they increased TNF- $\alpha$ production in the absence of LPS. ${ }^{24}$ However, the anti-inflammatory effect was more marked than the proinflammatory effect. ${ }^{24}$

Finally, we investigated the roles of the nanosize effect and coating material in the anti-inflammatory activity of PSCSPIONs. We found that DMSA-SPIONs decreased the secretion of inflammatory cytokines induced by $P$. gingivalis LPS, while PSC (not associated with SPIONs) did not (Figures 6 and 7). Therefore, the activity of PSC-SPIONs is likely related to the nanosize effect rather than to the coating material. Specifically, the anti-inflammatory effects of PSC-SPIONs are neither due to antibacterial properties nor the ability to reverse the inflammatory condition caused by LPS, but to their ability to prevent $P$. gingivalis invasion of hGFs and the competitive binding of $P$. gingivalis LPS and PSC-SPIONs to the same receptors on the hGF cell membrane. The nanosize of PSC-SPIONs may allow them to interact with cell membrane receptors such as TLR-4. Thus, all nanomaterials with good biosafety should have similar effects. Gold nanoparticles could be investigated in the future.

The current study is the first to show that SPIONs have anti-inflammatory effects in $P$. gingivalis-infected hGFs. However, our study had some limitations. Only in vitro studies were performed; the molecular pathway by which ROS levels are decreased was not explored; and the molecular anti-inflammatory mechanism by which PSCSPIONs suppress LPS-induced NF- $\kappa \mathrm{B}$ activation and I $\kappa \mathrm{B} \alpha$ phosphorylation was not clarified. We intend to address these questions in future studies.

\section{Conclusion}

PSC-SPIONs prevent $P$. gingivalis invasion of hGFs and inhibit $P$. gingivalis LPS-induced ROS production and upregulation of proinflammatory cytokines in hGFs. Although animal studies and preclinical tests are still 
needed, our results suggest that biocompatible PSCSPIONs could be applied clinically to solve the problem of $P$. gingivalis invasion and inflammatory stimulation in hGFs.

\section{Abbreviations}

PSC, polyglucose sorbitol carboxymethyl ether; SPIONs, superparamagnetic iron oxide nanoparticles; hGFs, human gingival fibroblasts; P. g, Porphyromonas gingivalis; LPS, lipopolysaccharide; ROS, reactive oxygen species; NF- $\kappa \mathrm{B}$, nuclear factor-kappa B; TLR, toll-like receptor; MOI, multiplicity of infection; BHI, brain-heart infusion; SEM, scanning electron microscopy; TEM, transmission electron microscopy; ELISA, enzyme-linked immunosorbent assay; qRT-PCR, quantitative reverse transcription-polymerase chain reaction.

\section{Acknowledgments}

The authors thank Chuanlin Sun from Shiyanjia Lab (www. shiyanjia.com) for SQUID analysis. This study was supported by grants from the Natural Science Foundation of Jiangsu Province (BK20211249) to Y Xia; the Open Project of State Key Laboratory (2019KA01) to Y Zhao; and Key Military Medical Projects (BLB20J001) to Y Zhao. Furthermore, the authors thank a Project Funded by the Priority Academic Program Development of Jiangsu Higher Education Institutions (PAPD, 2018-87) to Y Xia for their financial support. The authors have no other relevant affiliations or financial involvement with any organization or entity with a financial interest in or financial conflict with the subject matter or materials discussed in this manuscript apart from those disclosed. Writing assistance was provided by ELIXIGEN Co. Language Editing.

\section{Disclosure}

The authors report no conflicts of interest in this work.

\section{References}

1. Slots J. Periodontitis: facts, fallacies and the future. Periodontol. 2017;75(1):7-23. doi:10.1111/prd.12221

2. Eke PI, Borgnakke WS, Genco RJ. Recent epidemiologic trends in periodontitis in the USA. Periodontol. 2020;82(1):257-267. doi: $10.1111 /$ prd. 12323

3. Sanz M, Marco Del Castillo A, Jepsen S, et al. Periodontitis and cardiovascular diseases: consensus report. $J$ Clin Periodontol. 2020;47(3):268-288. doi:10.1111/jcpe.13189

4. Nascimento GG, Leite FRM, Vestergaard P, Scheutz F, López R. Does diabetes increase the risk of periodontitis? A systematic review and meta-regression analysis of longitudinal prospective studies. Acta Diabetol. 2018;55(7):653-667. doi:10.1007/s00592-018-1120-4
5. Ceccarelli F, Saccucci M, Di Carlo G, et al. Periodontitis and rheumatoid arthritis: the same inflammatory mediators? Mediators Inflamm. 2019;2019:6034546. doi:10.1155/2019/6034546

6. Murakami S, Mealey BL, Mariotti A, Chapple ILC. Dental plaqueinduced gingival conditions. J Clin Periodontol. 2018;45(Suppl 20): S17-S27. doi:10.1002/JPER.17-0095

7. Trombelli L, Farina R, Silva CO, Tatakis DN. Plaque-induced gingivitis: case definition and diagnostic considerations. J Periodontol. 2018;89(Suppl 1):S46-S73. doi:10.1002/JPER.17-0576

8. Ara T, Kurata K, Hirai K, et al. Human gingival fibroblasts are critical in sustaining inflammation in periodontal disease. $J$ Periodontal Res. 2009;44(1):21-27. doi:10.1111/j.16000765.2007.01041.x

9. Hao $\mathrm{C}, \mathrm{Wu} \mathrm{B}$, Hou Z, et al. Asiatic acid inhibits LPS-induced inflammatory response in human gingival fibroblasts. Int Immunopharmacol. $\quad 2017 ; 50: 313-318 . \quad$ doi:10.1016/j.intimp.20 17.07.005

10. Bhattarai G, Poudel SB, Kook SH, Lee JC. Resveratrol prevents alveolar bone loss in an experimental rat model of periodontitis. Acta Biomater. 2016;29:398-408. doi:10.1016/j.actbio.2015.10.031

11. Mysak J, Podzimek S, Sommerova P, et al. Porphyromonas gingivalis: major periodontopathic pathogen overview. J Immunol Res. 2014;2014:476068. doi:10.1155/2014/476068

12. Romano M, Uchiyama MK, Cardoso RM, Toma SH, Baptista MS, Araki K. Nitric oxide inhibition of lipopolysaccharide-stimulated RAW 247.6 cells by ibuprofen-conjugated iron oxide nanoparticles. Nanomedicine. 2020;15(25):2475-2492. doi:10.2217/nnm-20200214

13. Ho MH, Huang L, Goodwin JS, Dong X, Chen CH, Xie H. Two small molecules block oral epithelial cell invasion by Porphyromons gingivalis. PLoS One. 2016;11(2):e0149618. doi:10.1371/journal. pone. 0149618

14. Jia L, Han N, Du J, Guo L, Luo Z, Liu Y. Pathogenesis of important virulence factors of Porphyromonas gingivalis via toll-like receptors. Front Cell Infect Microbiol. 2019;9:262. doi:10.3389/ fcimb.2019.00262

15. Javed F, Salehpoor D, Al-Dhafeeri T, et al. Is adjunctive photodynamic therapy more effective than scaling and root planing alone in the treatment of periodontal disease in hyperglycemic patients? A systematic review. Photodiagnosis Photodyn Ther. 2018;22:1-6. doi:10.1016/j.pdpdt.2018.02.012

16. Jepsen K, Jepsen S. Antibiotics/antimicrobials: systemic and local administration in the therapy of mild to moderately advanced periodontitis. Periodontol. 2016;71(1):82-112. doi:10.1111/prd.12121

17. Wahajuddin AS. Superparamagnetic iron oxide nanoparticles: magnetic nanoplatforms as drug carriers. Int $J$ Nanomedicine. 2012;7:3445-3471. doi:10.2147/IJN.S30320

18. Wang G, Zhao J, Zhang M, et al. Ferumoxytol and CpG oligodeoxynucleotide 2395 synergistically enhance antitumor activity of macrophages against NSCLC with EGFRL858R/T790M mutation. Int J Nanomedicine. 2019;14:4503-4515. doi:10.2147/IJN.S193583

19. Li J, Feng Z, Gu N, et al. Superparamagnetic iron oxide nanoparticles assembled magnetic nanobubbles and their application for neural stem cells labeling. J Mater Sci Technol. 2021;4:124-132. doi:10.1016/j.jmst.2020.02.045

20. Wang Q, Chen B, Cao M, et al. Response of MAPK pathway to iron oxide nanoparticles in vitro treatment promotes osteogenic differentiation of hBMSCs. Biomaterials. 2016;86:11-20. doi:10.1016/j. biomaterials.2016.02.004

21. Xia Y, Guo Y, Yang Z, et al. Iron oxide nanoparticle-calcium phosphate cement enhanced the osteogenic activities of stem cells through WNT/ $\beta$-catenin signaling. Mater Sci Eng C Mater Biol Appl. 2019;104:109955. doi:10.1016/j.msec.2019.109955

22. Xu Y, Li Y, Liu X, et al. SPIONs enhances IL-10-producing macrophages to relieve sepsis via Cav1-Notch1/HES1-mediated autophagy. Int J Nanomedicine. 2019;14:6779-6797. doi:10.2147/IJN.S215055 
23. Yu P, Zheng L, Wang P, et al. Development of a novel polysaccharidebased iron oxide nanoparticle to prevent iron accumulation-related osteoporosis by scavenging reactive oxygen species. Int J Biol Macromol. 2020;165(Pt B):1634-1645. doi:10.1016/j.ijbiomac.2020.10.016

24. Chen Y, Zeng Z, Ying H, Wu C, Chen S. Superparamagnetic iron oxide nanoparticles attenuate lipopolysaccharide-induced inflammatory responses through modulation of toll-like receptor 4 expression. J Appl Toxicol. 2020;40(8):1067-1075. doi:10.1002/jat.3967

25. Grosse S, Stenvik J, Nilsen AM. Iron oxide nanoparticles modulate lipopolysaccharide-induced inflammatory responses in primary human monocytes. Int $J$ Nanomedicine. 2016;11:4625-4642. doi:10.2147/IJN.S113425

26. Kang W, Shang L, Wang T, Liu H, Ge S. Rho-kinase inhibitor Y-27632 downregulates LPS-induced IL- 6 and IL- 8 production via blocking p38 MAPK and NF- $\mathrm{B}$ pathways in human gingival fibroblasts. $J$ Periodontol. 2018;89(7):883-893. doi:10.1002/JPER.17-0571

27. Chen B, Li Y, Zhang X, et al. An efficient synthesis of ferumoxytol induced by alternating-current magnetic field. Mater Lett. 2016;170:93-96. doi:10.1016/j.matlet.2016.02.006

28. Zou J, Zhang Y, Sun J, et al. Deoxyelephantopin induces reactive oxygen species-mediated apoptosis and autophagy in human osteosarcoma cells. Cell Physiol Biochem. 2017;42(5):1812-1821. doi: $10.1159 / 000479537$

29. Yang Q, Liu S, Liu X, Liu Z, Xue W, Zhang Y. Role of chargereversal in the hemo/immuno-compatibility of polycationic gene delivery systems. Acta Biomater. 2019;96:436-455. doi:10.1016/j. actbio.2019.06.043

30. Pan C, Liu J, Wang H, Song J, Tan L, Zhao H. Porphyromonas gingivalis can invade periodontal ligament stem cells. $B M C$ Microbiol. 2017;17(1):38. doi:10.1186/s12866-017-0950-5

31. Li X, Wang X, Zheng M, Luan QX. Mitochondrial reactive oxygen species mediate the lipopolysaccharide-induced pro-inflammatory response in human gingival fibroblasts. Exp Cell Res. 2016;347 (1):212-221. doi:10.1016/j.yexcr.2016.08.007

32. Zhang L, Wang X, Zou J, Liu Y, Wang J. DMSA-coated iron oxide nanoparticles greatly affect the expression of genes coding cysteinerich proteins by their DMSA coating. Chem Res Toxicol. 2015;28 (10):1961-1974. doi:10.1021/acs.chemrestox.5b00161

33. Han C, Shen H, Yang Y, et al. Antrodia camphorata polysaccharide resists 6-OHDA-induced dopaminergic neuronal damage by inhibiting ROS-NLRP3 activation. Brain Behav. 2020;10(11):e01824. doi:10.1002/brb3.1824

34. Chen B, Sun J, Fan F, et al. Ferumoxytol of ultrahigh magnetization produced by hydrocooling and magnetically internal heating co-precipitation. Nanoscale. 2018;10(16):7369-7376. doi:10.1039/ c8nr00736e
35. Wang G, Serkova NJ, Groman EV, Scheinman RI, Simberg D. Feraheme (Ferumoxytol) is recognized by proinflammatory and anti-inflammatory macrophages via scavenger receptor Type AI/II. Mol Pharm. 2019;16 (10):4274 4281. doi:10.1021/acs.molpharmaceut.9b00632

36. Wang P, Ma S, Ning G, et al. Entry-prohibited effect of $\mathrm{kHz}$ pulsed magnetic field upon interaction between SPIO nanoparticles and mesenchymal stem cells. IEEE Trans Biomed Eng. 2020;67 (4):1152-1158. doi:10.1109/TBME.2019.2931774

37. Boisvert H, Duncan MJ. Clathrin-dependent entry of a gingipain adhesin peptide and Porphyromonas gingivalis into host cells. Cell Microbiol. 2008;10(12):2538-2552. doi:10.1111/j.14625822.2008.01228.x

38. Kanzaki H, Wada S, Narimiya T, et al. Pathways that regulate ROS scavenging enzymes, and their role in defense against tissue destruction in periodontitis. Front Physiol. 2017;8:351. doi:10.3389/ fphys.2017.00351

39. Huang C, Zhang C, Yang P, et al. Eldecalcitol inhibits LPS-induced NLRP3 inflammasome-dependent pyroptosis in human gingival fibroblasts by activating the Nrf2/HO-1 signaling pathway. Drug Des Devel Ther. 2020;14:4901-4913. doi:10.2147/DDDT.S269223

40. Rostami AA, Mohseni Kouchesfahani H, Kiani S, Fakheri R. Iron oxide nanoparticles reduced retinoic acid induced-neuronal differentiation of mouse embryonic stem cells by ROS generation. Arch Iran Med. 2015;18(9):586-590.

41. Wen T, Du L, Chen B, et al. Iron oxide nanoparticles induce reversible endothelial-to-mesenchymal transition in vascular endothelial cells at acutely non-cytotoxic concentrations. Part Fibre Toxicol. 2019;16(1):30. doi:10.1186/s12989-019-0314-4

42. Xu Y, Xue Y, Liu X, et al. Ferumoxytol attenuates the function of MDSCs to ameliorate LPS-induced immunosuppression in sepsis. Nanoscale Res Lett. 2019;14(1):379. doi:10.1186/s11671-019-3209-2

43. Le Sage F, Meilhac O, Gonthier MP. Porphyromonas gingivalis lipopolysaccharide induces pro-inflammatory adipokine secretion and oxidative stress by regulating Toll-like receptor-mediated signaling pathways and redox enzymes in adipocytes. Mol Cell Endocrinol. 2017;446:102-110. doi:10.1016/j.mce.2017.02.022

44. Jin R, Liu L, Zhu W, et al. Iron oxide nanoparticles promote macrophage autophagy and inflammatory response through activation of toll-like Receptor-4 signaling. Biomaterials. 2019;203:23-30. doi:10.1016/j.biomaterials.2019.02.026

45. Mulens-Arias V, Rojas JM, Pérez-Yagüe S, Morales MP, Barber DF. Polyethylenimine-coated SPIONs trigger macrophage activation through TLR-4 signaling and ROS production and modulate podosome dynamics. Biomaterials. 2015;52:494-506. doi:10.1016/j. biomaterials.2015.02.068
International Journal of Nanomedicine

\section{Publish your work in this journal}

The International Journal of Nanomedicine is an international, peerreviewed journal focusing on the application of nanotechnology in diagnostics, therapeutics, and drug delivery systems throughout the biomedical field. This journal is indexed on PubMed Central, MedLine, CAS, SciSearch ${ }^{\mathbb{B}}$, Current Contents ${ }^{\mathbb{B}} /$ Clinical Medicine, $^{2}$
Journal Citation Reports/Science Edition, EMBase, Scopus and the Elsevier Bibliographic databases. The manuscript management system is completely online and includes a very quick and fair peer-review system, which is all easy to use. Visit http://www.dovepress.com/ testimonials.php to read real quotes from published authors. 